STRUCTURAL BIOLOGY COMMUNICATIONS

ISSN 2053-230X

Received 7 July 2020

Accepted 18 August 2020

Edited by M. A. Hough, University of Essex, United Kingdom

Keywords: inositol monophosphatase; IMPase; ebselen; bipolar disorder; tetramer.

PDB reference: human IMPase bound to ebselen, 6zk0

Supporting information: this article has supporting information at journals.iucr.org/f

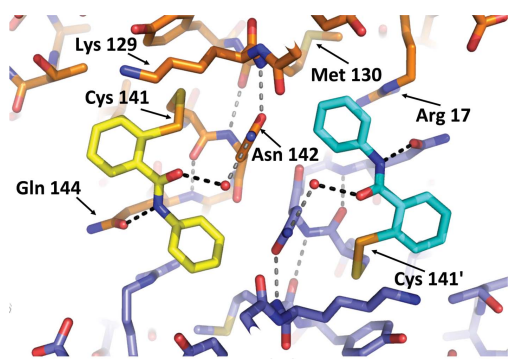
OPEN $\odot$ ACCESS

\section{Crystallization and structure of ebselen bound to Cys141 of human inositol monophosphatase}

\author{
Gareth D. Fenn,* Helen Waller-Evans, John R. Atack and Benjamin D. Bax* \\ Medicines Discovery Institute, School of Biosciences, Cardiff University, Cardiff CF10 3AT, United Kingdom. \\ *Correspondence e-mail: fenngd@cardiff.ac.uk, baxb@cardiff.ac.uk
}

Inositol monophosphatase (IMPase) is inhibited by lithium, which is the most efficacious treatment for bipolar disorder. Several therapies have been approved, or are going through clinical trials, aimed at the replacement of lithium in the treatment of bipolar disorder. One candidate small molecule is ebselen, a selenium-containing antioxidant, which has been demonstrated to produce lithium-like effects both in a murine model and in clinical trials. Here, the crystallization and the first structure of human IMPase covalently complexed with ebselen, a $1.47 \AA$ resolution crystal structure (PDB entry 6zk0), are presented. In the complex with human IMPase, ebselen in a ring-opened conformation is covalently attached to Cys141, a residue located away from the active site. IMPase is a dimeric enzyme and in the crystal structure two adjacent dimers share four ebselen molecules, creating a tetramer with approximate 222 symmetry. In the crystal structure presented in this publication, the active site in the tetramer is still accessible, suggesting that ebselen may function as an allosteric inhibitor or may block the binding of partner proteins.

\section{Introduction}

Bipolar disorder is a chronic and debilitating psychiatric disorder that is characterized by cycles of mania followed by severe depression, frequently accompanied by bouts of psychosis. Although antipsychotic agents are the preferred short-term method of treatment, more efficacious moodstabilizing drugs, such as lithium, are used in long-term clinical management (Geddes \& Miklowitz, 2013). Lithium is the gold-standard treatment for bipolar disorder; however, it has several serious side effects, such as nausea and cognitive impairment, in addition to a narrow therapeutic window (Rybakowski, 2016). Because of these liabilities, other less efficacious mood stabilisers (for example lamotrigine) are now often used in the treatment of bipolar disorder (Won \& Kim, 2017). One enzyme inhibited by lithium is inositol monophosphatase (IMPase; Gill et al., 2005), which has led to rational drug design targeting IMPase as a strategy for developing novel therapies for bipolar disorder (Brown \& Tracy, 2013).

IMPase is a key enzyme in the intracellular phosphatidylinositol (PI) signalling pathway, whereby IMPase dephosphorylates inositol 1-, 3- or 4-phosphate, collectively known as InsP1, to produce myo-inositol, also known as free inositol (Atack et al., 1995). Cleavage of InsP1 into myo-inositol by IMPase is required for the recycling of inositol for subsequent use in the PI signalling pathway (Atack et al., 1995). Inositol is an essential precursor for the synthesis of PI, which is subsequently utilized in the synthesis of phosphatidylinositol phosphates (PIPs). These include $\mathrm{PI}(4,5) \mathrm{P}_{2}$, which is cleaved 
by phospholipase $\mathrm{C}$ following GPCR signalling to release the second messengers diacylglycerol (DAG) and inositol 1,4,5trisphosphate $\left(\mathrm{IP}_{3}\right)$ (Phiel \& Klein, 2001). The observed depletion of free inositol and accumulation of the substrate of IMPase, InsP1, coupled with a reduction in agonist-invoked $\mathrm{IP}_{3}$ formation, in cells and animals treated with lithium led to the development of the inositol-depletion hypothesis to explain the mechanism by which lithium exerts its effects (Berridge et al., 1989).

The inositol-depletion hypothesis suggests that lithium produces a reduction in free inositol primarily via blocking the recycling of inositol from InsP1, which leads to a decrease in $\mathrm{PI}(4,5) \mathrm{P}_{2}$ and a slowing of the PIP signalling pathways that are postulated to be hyperactive in bipolar disorder (Harwood, 2005). Further evidence to support the inositol-depletion hypothesis comes from observations that the mood stabilisers carbamazepine and valproic acid also lead to the depletion of free inositol and attenuation of $\mathrm{PI}(4,5) \mathrm{P}_{2}$ signalling pathways (Williams et al., 2002). Therefore, targeting IMPase as a means of depleting free inositol is of scientific interest and led to the search for new IMPase inhibitors.

One such IMPase inhibitor is ebselen [2-phenyl-1,2-benzisoselenazol- $3(2 \mathrm{H})$-one], an organoselenium compound which functions as a glutathione peroxidase mimic (Nakamura et al., 2002). Ebselen is believed to act through the reduction of reactive oxygen species (ROS) by binding covalently to cysteine residues or thiols to form selenosulfide bonds that lead to its pharmacological effect (Azad \& Tomar, 2014). However, it is not known whether the covalent binding of ebselen to specific groups is directly or indirectly responsible for its mechanism of action (Ullrich et al., 1996).

Ebselen has been demonstrated to inhibit IMPase in a covalent manner, with effects consistent with those of lithium, through depletion of free inositol in mouse brain (Singh et al., 2013). Subsequent trials in a healthy cohort demonstrated that ebselen leads to decreased myo-inositol in the anterior cingulate cortex, in addition to effects consistent with the attenuation of PIP signalling (Singh et al., 2015). At present ebselen is currently in a stage 2 clinical trial for the treatment of bipolar disorder; however, results from the trial have not been released at the time of writing.

Ebselen is known to bind to several proteins; crystal structures show ebselen covalently bound to cysteine residues of proteins including SOD1 (Capper et al., 2018; Chantadul et al., 2020) and the transpeptidase LdtMt2 from Mycobacterium tuberculosis (de Munnik et al., 2019). Ebselen has also been reported to inhibit the main protease from SARS-CoV-2 (Jin et al., 2020). These multiple targets suggest several potential therapeutic uses for ebselen, but also that there are likely to be off-target side effects.

Whilst inhibition of IMPase by ebselen has been demonstrated in vitro, confirmation of the ebselen binding loci and the exact mechanism of action on IMPase remain unclear. Structures of IMPase have been published with a variety of ligands, including a structure of human IMPase with the lithium mimetic L-690,330 (Kraft et al., 2018). In this paper, we present a $1.47 \AA$ resolution structure of ebselen covalently
Table 1

Macromolecule-production information.

\begin{tabular}{|c|c|}
\hline Source organism & Human \\
\hline DNA source & $\begin{array}{l}\text { pET-15b vector containing human inositol } \\
\text { monophosphatase with an N-terminal } \\
\text { His }{ }_{6} \text { tag followed by an HRV } 3 \mathrm{C} \\
\text { cleavage site }\end{array}$ \\
\hline Cloning vector & pET-15b \\
\hline Expression vector & pET-15b \\
\hline Expression host & E. coli Rosetta2 (DE3) \\
\hline $\begin{array}{l}\text { Complete amino-acid sequence } \\
\text { of the construct produced }\end{array}$ & $\begin{array}{l}\text { MHHHHHHLEVLFQGPMADPWQECMDYAVTL } \\
\text { ARQAGEVVCEAIKNEMNVMLKSSPVDLV } \\
\text { TATDQKVEKMLISSIKEKYPSHSFIGEE } \\
\text { SVAAGEKSILTDNPTWIIDPIDGTTNFV } \\
\text { HRFPFVAVSIGFAVNKKIEFGVVYSCVE } \\
\text { GKMYTARKGKGAFCNGQKLQVSQEDIT } \\
\text { KSLLVTELGSSRTPETVMVLSNMEKLF } \\
\text { CIPVHGIRSVGTAAVNMCLVATGGADAY } \\
\text { YEMGIHCWDVAGAGIIVTEAGGVLMDVT } \\
\text { GGPFDLSRRVIAANNRILAERIAKEIQ } \\
\text { VIPLQRDDED }\end{array}$ \\
\hline
\end{tabular}

bound to cysteine residue 141 of human IMPase (PDB entry $6 \mathrm{zk} 0$ ). This is the first structure of IMPase to be published that demonstrates direct covalent binding of ebselen to IMPase.

\section{Materials and methods}

All reagents were purchased from Sigma-Aldrich or Thermo Fisher unless stated otherwise.

\subsection{Macromolecule production}

The IMPase construct was as described by Kraft et al. (2018) and Escherichia coli Rosetta2 (DE3) cells were used for IMPase production. A starter culture $(10 \mathrm{ml})$ of transformed E. coli cells was grown overnight and was used to inoculate 11 LB medium containing $2.5 \mathrm{~m} M$ betaine, $660 \mathrm{~m} M$ sorbitol, $35 \mathrm{mg} \mathrm{ml}^{-1}$ chloramphenicol and $50 \mathrm{mg} \mathrm{ml}^{-1}$ ampicillin. The culture was grown at $37^{\circ} \mathrm{C}$ to an $\mathrm{OD}_{600}$ of 0.8 . IMPase expression was induced by the addition of $0.5 \mathrm{~m} M$ IPTG and the culture was grown overnight at $25^{\circ} \mathrm{C}$.

The E. coli cells were pelleted by centrifugation at $9600 \mathrm{~g}$ for $20 \mathrm{~min}$ at $4^{\circ} \mathrm{C}$ and were resuspended in $50 \mathrm{ml}$ lysis buffer [20 m $M$ Tris- $\mathrm{HCl} \mathrm{pH} 7.8,150 \mathrm{~m} M \mathrm{NaCl}, 40 \mathrm{U} \mathrm{ml}^{-1}$ DNAse and one EDTA-free protease-inhibitor tablet (Roche)]. The resuspended pellet was lysed by sonication and the debris was pelleted by centrifugation at $33000 \mathrm{~g}$ for $20 \mathrm{~min}$ at $4^{\circ} \mathrm{C}$. The clarified sonicate was heat-treated at $68^{\circ} \mathrm{C}$ for $1 \mathrm{~h}$ and the precipitant was pelleted by centrifugation at $32000 \mathrm{~g}$ for $20 \mathrm{~min}$ at $4^{\circ} \mathrm{C}$. The supernatant was then incubated with $\mathrm{Co}-$ NTA resin at $4^{\circ} \mathrm{C}$ for $1 \mathrm{~h}$. After incubation, the resin was washed with seven resin volumes (RV) of wash buffer ( $20 \mathrm{mM}$ Tris- $\mathrm{HCl} \mathrm{pH} 7.8,150 \mathrm{~m} M \mathrm{NaCl}, 15 \mathrm{~m} M$ imidazole) and the IMPase was eluted with $5 \mathrm{RV}$ of elution buffer $(20 \mathrm{~m} M$ Tris$\mathrm{HCl} \mathrm{pH} 7.8,150 \mathrm{~m} M \mathrm{NaCl}, 250 \mathrm{~m} M$ imidazole).

The eluted fractions were pooled and incubated overnight at $4^{\circ} \mathrm{C}$ with Pierce HRV $3 \mathrm{C}$ protease solution. This removes the N-terminal tag (MHHHHHHLEVLFQ) by cleaving at LEVLFQ $\downarrow$ GP (the sequence is given in Table 1). Cleaved IMPase was purified initially by incubation with Glutathione 
Table 2

Crystallization conditions.

\begin{tabular}{ll}
\hline Method & Sitting-drop vapour diffusion \\
Plate type & SWISSCI 3 Lens crystallization plate \\
Temperature $(\mathrm{K})$ & 293 \\
Protein concentration $\left(\mathrm{mg} \mathrm{ml}^{-1}\right)$ & 20 \\
Buffer composition of protein & $20 \mathrm{~m} M$ Tris- $\mathrm{HCl} \mathrm{pH} 7.8,150 \mathrm{~m} \mathrm{M} \mathrm{NaCl}$, \\
$\quad$ solution & $1 \mathrm{~m} M$ EDTA, 10\% $(v / v)$ glycerol \\
Composition of reservoir & $0.2 \mathrm{M} \mathrm{MnSO}, 0.1 \mathrm{M} \mathrm{MES}, 28 \%$ PEG 4000 \\
$\quad$ solution & $\mathrm{pH} \mathrm{5.5}$ \\
Volume and ratio of drop & $100 \mathrm{nl}, 1: 1$ \\
Volume of reservoir $(\mu \mathrm{l})$ & 50 \\
\hline
\end{tabular}

Sepharose 4 Fast Flow Resin that had been pre-equilibrated with two column volumes of size-exclusion chromatography (SEC) buffer (20 mM Tris- $\mathrm{HCl} \mathrm{pH} 7.8,150 \mathrm{mM} \mathrm{NaCl}$ ). The flowthrough from the column was collected, concentrated to $5 \mathrm{ml}$ and loaded onto a HiLoad 26/600 Superdex 75 PrepGrade SEC column pre-equilibrated with SEC buffer. The proteins were gel-filtered at a flow rate of $1 \mathrm{ml} \mathrm{min}^{-1}$ and the fractions containing IMPase were pooled, buffer-exchanged into storage buffer [20 mM Tris- $\mathrm{HCl}$ pH 7.8, $150 \mathrm{mM} \mathrm{NaCl}$, $1 \mathrm{~m} M$ EDTA, $10 \%(v / v)$ glycerol] and concentrated to $20 \mathrm{mg} \mathrm{ml}^{-1}$ using $10 \mathrm{kDa}$ cutoff protein concentrators at $4000 \mathrm{~g}$ prior to storage at $-20^{\circ} \mathrm{C}$. This protocol gave a typical yield of $2 \mathrm{mg}$ IMPase per litre of culture.

\subsection{Crystallization}

The crystallization of human IMPase was carried out using SWISSCI 3 Lens sitting-drop plates; the plates were set up using a Mosquito liquid-handling robot (TTP Labtech). To co-crystallize IMPase with ebselen, a $20 \mu \mathrm{l}$ aliquot of IMPase at $20 \mathrm{mg} \mathrm{ml}^{-1}$ in storage buffer [20 $\mathrm{m} M$ Tris- $\mathrm{HCl} \mathrm{pH} \mathrm{7.8,}$ $150 \mathrm{~m} M \mathrm{NaCl}, 1 \mathrm{~m} M$ EDTA, $10 \%(v / v)$ glycerol] was incubated with $50 \mathrm{~m} M$ ebselen $(6 \mu \mathrm{l}$ of $200 \mathrm{mM}$ ebselen in DMSO). The sample was transferred to a $500 \mu \mathrm{l}$ microcentrifuge tube, placed on a roller and incubated at room temperature for $30 \mathrm{~min}$ prior to setting up crystallization plates.

A reservoir solution consisting of $0.2 M \mathrm{MnSO}_{4}, 0.1 \mathrm{M}$ MES, 28\% PEG $4000 \mathrm{pH} 5.5$ was added to the IMPase and ebselen solution in a $1: 1$ ratio and incubated at $20^{\circ} \mathrm{C}$ (see Table 2). Crystals appeared after seven days and continued to grow until they were harvestesd for data collection on day 14; they were cryoprotected by transfer into $20 \%(v / v)$ glycerol, $80 \%(v / v)$ reservoir solution and flash-cooled prior to data collection.

\subsection{Data collection and processing}

An X-ray diffraction data set was collected from a single cryocooled crystal on beamline I04-1 at Diamond Light Source (Table 3). The data (2000 images of $0.1^{\circ}$ ) were processed with the xia2 pipeline at Diamond Light Source (Winter, 2010; Winter et al., 2013) to give a $1.47 \AA$ resolution data set in space group $P 3_{1} 21$, with unit-cell parameters $a=b=84.02, c=150.22 \AA, \alpha=\beta=90, \gamma=120^{\circ}$.

There are nine crystal structures of human IMPase in the PDB with similar unit-cell parameters, all of which belong
Table 3

Data collection and processing.

Values in parentheses are for the outer shell. Test data sets were also produced, merging the first 800 and last 800 images from the data set, to check for radiation-damage effects (see Section 2.4 for details). The analysis suggested that the best data set was obtained by using all data, and that although some radiation damage appeared to be present in the data, this damage was not reduced by removing the later frames from the data set.
Diffraction source

Wavelength $(\AA)$

Temperature (K)

Detector

Crystal-to-detector distance (mm)

Rotation range per image $\left(^{\circ}\right)$

Total rotation range $\left({ }^{\circ}\right)$

Exposure time per image (s)

Space group

$a, b, c(\AA)$

$\alpha, \beta, \gamma\left({ }^{\circ}\right)$

Mosaicity $\left(^{\circ}\right)$

Resolution range $(\AA)$

Total No. of reflections

No. of unique reflections

Completeness (\%)

Multiplicity

$\langle I / \sigma(I)\rangle$

$\mathrm{CC}_{1 / 2}$

$R_{\text {meas }}$

$R_{\text {p.im. }}$

Overall $B$ factor from Wilson plot $\left(\AA^{2}\right)$
I04-1, Diamond Light Source 0.91188

100

PILATUS 6M-F

260.0

0.1

200

0.1

$P 3{ }_{2} 21$

$84.02,84.02,150.22$

$90.0,90.0,120.0$

0.080

45.0-1.47 (1.50-1.47)

$1155862(53779)$

$104886(5118)$

$100.0(100.0)$

$11.0(10.5)$

$20.4(1.2)$

$1.0(0.498)$

$0.056(2.123)$

$0.017(0.652)$

22.2 to space group $P 3_{2} 21$; thus, the data were re-indexed and remerged in $P 3_{2} 21$. The data were merged using AIMLESS version 0.7.4 (Evans \& Murshudov, 2013). The Rcp statistic, which is used to estimate cumulative radiation damage in AIMLESS (Diederichs, 2006), did not increase significantly over the 2000 frames.

\subsection{Structure solution and refinement}

The structure was solved by rigid-body refinement using the $1.7 \AA$ resolution crystal structure of IMPase with manganese (PDB entry 6gj0; Kraft et al., 2018). Initial structure solution used the DIMPLE pipeline; this pipeline provides the user with a quick method to identify data sets that have a bound ligand or drug candidate in the crystal (http://ccp4.github.io/ dimple/; Wojdyr et al., 2013).

Initial maps showed clear electron density (Fig. 1) for a single ebselen molecule attached to Cys141 in both subunits $A$ and $B$ of the dimer (the $P 3_{2} 21$ cell has one dimer in the asymmetric unit). The ebselen was built onto Cys141 $A$ and Cys141B in Coot (Emsley et al., 2010; Emsley, 2017). Restraints for the covalently bound ebselen were generated in AceDRG (Long et al., 2017) and the structure was refined using REFMAC5 (Murshudov et al., 2011).

Although the electron density was very clear for the terminal phenyl ring of the compound (Figs. $1 c$ and $1 d$ ) and the electron-density maps have a large peak for the $\mathrm{Se}$ atom (covalently bonded to the S atom of Cys141), the electron density suggests that some radiation damage has occurred to the sulfur-selenium bond (Weik et al., 2002; Garman, 2010). The data are consistent with a model in which some initial radiation damage occurred to the sulfur-selenium bond 
(within the first few degrees of data), after which a steady state occurred (the bond reforming after breaking owing to radiation; Gerstel et al., 2015).

Each active site contains three $\mathrm{Mn}^{2+}$ ions in PDB entry $6 \mathrm{gj} 0$ (Kraft et al., 2018), while in PDB entry 2bji (Gill et al., 2005) each active site contains three $\mathrm{Mg}^{2+}$ ions. In our structure, site 2 (Gill et al., 2005) does not have sufficient electron density for an $\mathrm{Mn}^{2+}$ ion $\left(\mathrm{Mn}^{2+}\right.$ ions have 23 electrons). We modelled a similarly coordinated sodium ion at this site, because we had no $\mathrm{Mg}^{2+}$ ions in our crystallization experiment (the protein was provided in $150 \mathrm{mM} \mathrm{NaCl}$ and the crystallization buffer contained $200 \mathrm{mM} \mathrm{MnSO} 4$ ). However, we cannot rule out the possibility that this is an $\mathrm{Mg}^{2+}$ ion, rather than an $\mathrm{Na}^{+}$ion (both $\mathrm{Na}^{+}$and $\mathrm{Mg}^{2+}$ ions have ten electrons). This 'site 2' is the position in which lithium is postulated to bind with tetrahedral coordination geometry (Gill et al., 2005). However, the co- ordination geometry in our structure is consistent with two $\mathrm{Mn}^{2+}$ ions and one $\mathrm{Mg}^{2+}$ ion, each with 'standard' octahedral coordination geometry. Most of the active-site metal atoms are modelled in two positions and have temperature factors similar to those of the surrounding residues (Masmaliyeva \& Murshudov, 2019).

In the deposited structure (PDB entry 6zk0), the ebselens on Cys141 in subunits $A$ and $B$ each have an occupancy of 0.6 , but the Se atoms are modelled in two positions (Supplementary Fig. S1). In the crystal, the selenium-sulfur bond has been modelled with an occupancy of 0.4 or 0.35 (see Supplementary Fig. S1). A second selenium position observed in the electrondensity maps is some $1.3 \AA$ further away from the $\mathrm{S}$ atom of Cys141 and this second position is likely to be caused by radiation-induced cleavage of the selenosulfide bond (Gerstel et al., 2015). Refinement statistics are summarized in Table 4.

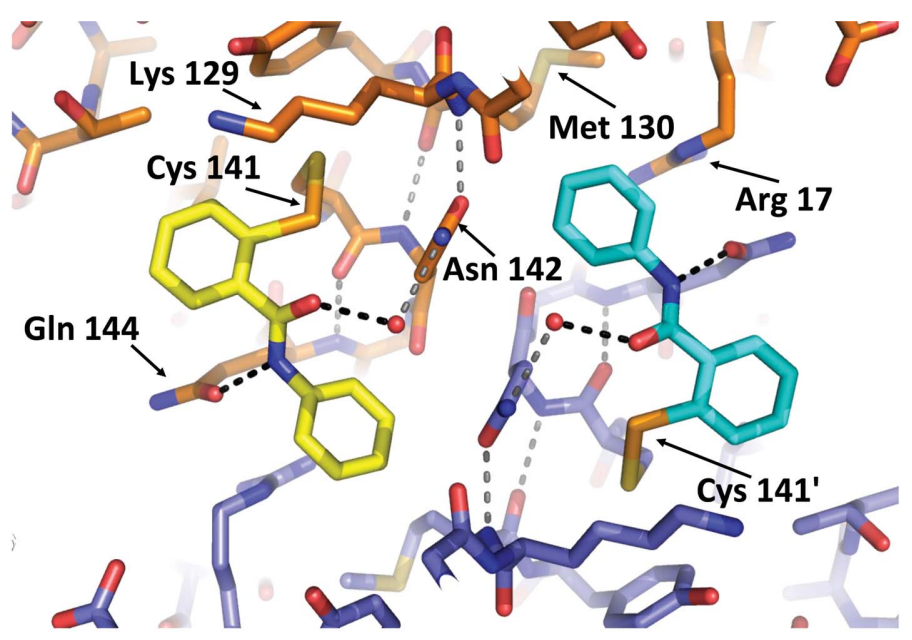

(a)<smiles>O=c1c2ccccc2[se]n1-c1ccccc1</smiles><smiles>O=C(Nc1ccccc1)c1ccccc1[Se]Sc1ccccc1</smiles>

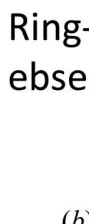

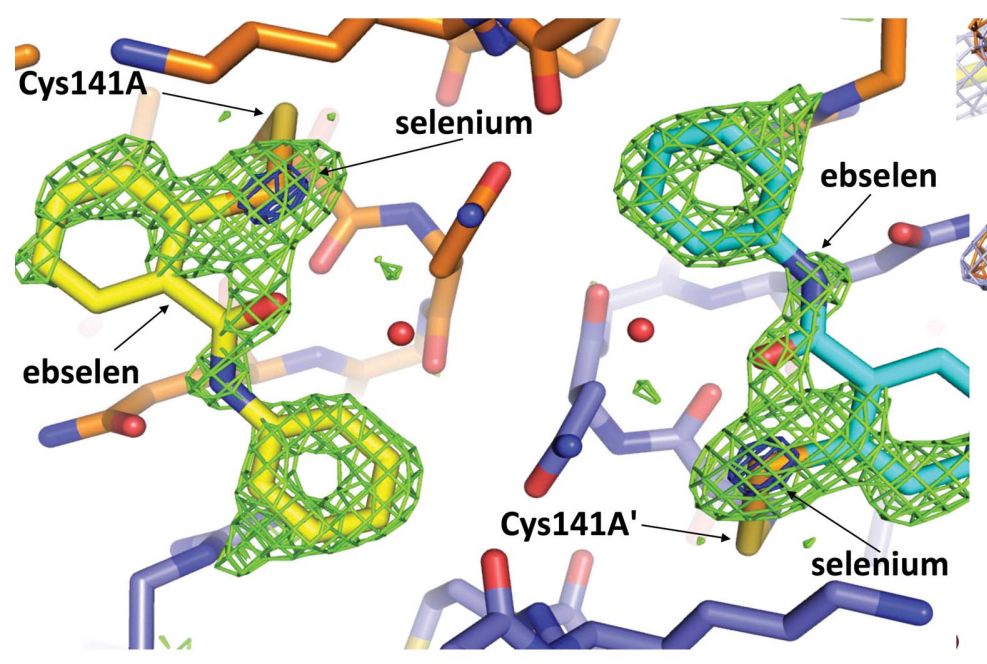

(c) (b)

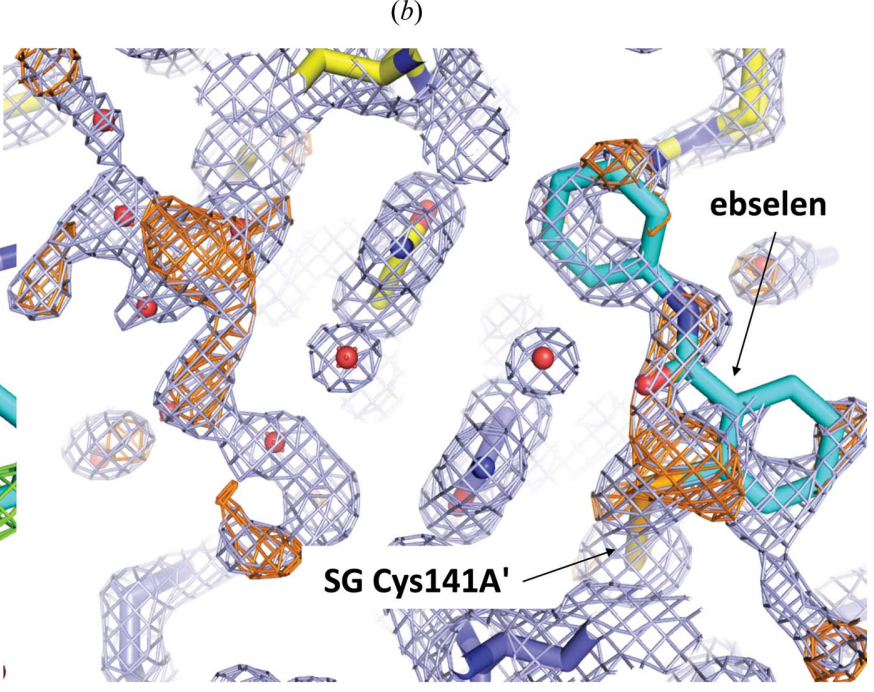

(d)

Figure 1

View of ebselen attached to Cys141 (PDB entry 6zk0). (a) Overview of two ebselen molecules attached to the $A$ and $A^{\prime}$ (symmetry-related) subunits around the crystallographic twofold axis. One subunit $(A)$ has $\mathrm{C}$ atoms in orange and the second subunit $\left(A^{\prime}\right)$ has $\mathrm{C}$ atoms in slate blue ( $\mathrm{N}$ atoms are blue, $\mathrm{O}$ atoms are red, Se atoms are orange and $\mathrm{S}$ atoms are yellow-orange). $\mathrm{C}$ atoms in one ebselen are yellow and those in the second ebselen are cyan. Hydrogen bonds near ebselen are indicated by dotted lines. (b) Chemical structures of ebselen and ring-opened ebselen on Cys141 (drawn with Marvin; https://www.chemaxon.com). (c) Final ebselen OMIT map $\left(F_{\mathrm{o}}-F_{\mathrm{c}} ; 3 \sigma\right.$, green; $15 \sigma$, blue). Note that the peaks on the seleniums (blue mesh) are $20.5 \sigma$ and $19.6 \sigma$ in this ebselen OMIT map. (d) Original DIMPLE (Wojdyr et al., 2013) $2 F_{\mathrm{o}}-F_{\mathrm{c}}$ map (1 $\sigma$, light blue) and $F_{\mathrm{o}}-F_{\mathrm{c}}$ difference map ( $3 \sigma$, orange). For subunit $A$ the DIMPLE-refined structure with waters (small red spheres) refined into the density for the ebselen is shown. For the $A^{\prime}$ subunit the 'final' coordinates (including ebselen) are shown. 


\section{Results}

\subsection{Human IMPase structure with ebselen bound at Cys141}

The $1.47 \AA$ resolution crystal structure of human IMPase with ebselen (PDB entry 6zk0) was solved using a structure of human IMPase with the same unit cell and space group (PDB entry 6gj0; Kraft et al., 2018). Electron-density maps (Fig. 1) clearly showed ebselen attached only to a single cysteine, Cys141. The binding of ebselen to the Cys141 residue in each monomer does not lead to noticeable changes in conformation in the active site that would prevent the catalytic activity of IMPase. Additionally, the binding of ebselen to Cys141 does not appear to prevent dimer formation, as shown by the dimers (and tetramers) present in this structure (PDB entry 6zk0). However, whilst this structure clearly shows ebselen bound to Cys141 in each monomer of IMPase, our structure does not rule out the possibility that Cys 218 could be modified in vivo.

IMPase contains seven cysteine residues, amino acids 8, 24, 125, 141, 184, 201 and 218; of these residues, only Cys 218 is near the active site. Four of the cysteine residues are buried and would not be expected to be accessible to modification by ebselen (Cys8, Cys125, Cys201 and Cys218). Of the three cysteines that have some surface accessibility in the monomer, one of them, Cys184, is largely buried in the dimer interface, as shown in Fig. 2 (PDB entry 6zk0). The procedure used to co-crystallize ebselen with IMPase allowed partial oxidation to form a Cys141-ebselen selenosulfide bond (Fig. 1). In our structure Cys24 has some surface accessibility when reduced, but when oxidized forms a disulfide with Cys125 (Fig. 2). Cys125 has no surface accessibility whether oxidized or

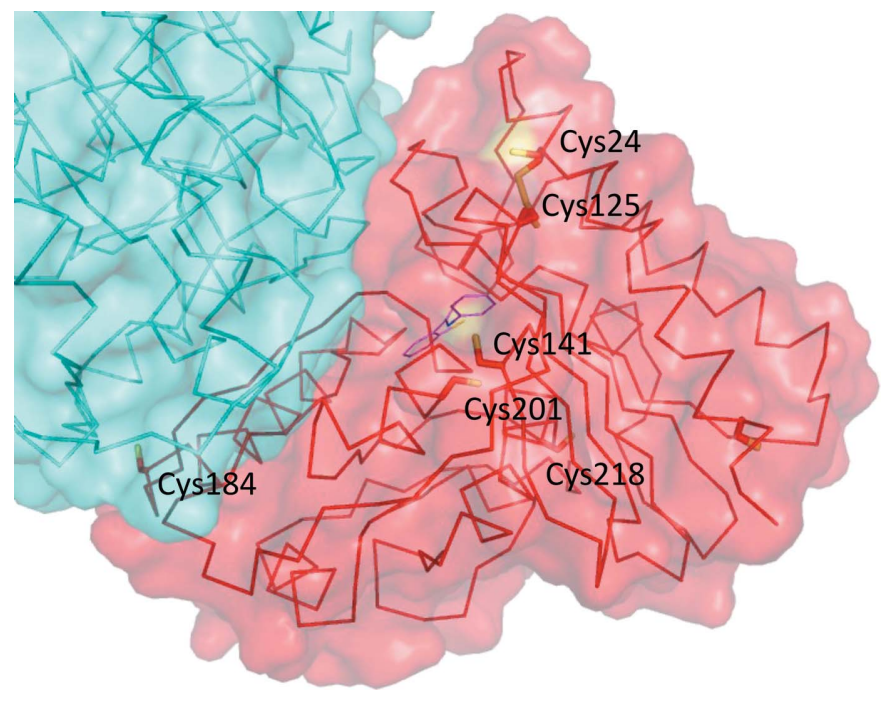

Figure 2

Cysteine residues in IMPase with ebselen bound to Cys141 (PDB entry 6zk0). IMPase is shown as a $\mathrm{C}^{\alpha}$ ribbon trace and the side chains of the seven cysteines are shown as sticks on the 'red' subunit. The second subunit in the dimer is shown in cyan. A semi-transparent surface is shown; note that where the $\mathrm{S}$ atoms of the cysteine residues are on the surface of the protein, the surface is yellow (Cys24 and Cys141). Cys184 also has some surface accessibility in the monomer, but is largely buried at the dimer interface, so no yellow is visible for Cys184 in this figure.
Table 4

Structure solution and refinement.

Values in parentheses are for the outer shell.

\begin{tabular}{ll}
\hline Resolution range $(\AA)$ & $24.44-1.47(1.50-1.47)$ \\
Completeness (\%) & $100.0(100.0)$ \\
$\sigma$ Cutoff & N/A \\
No. of reflections, working set & 99740 \\
No. of reflections, test set & 5081 \\
Final $R_{\text {cryst }}$ & 0.1734 \\
Final $R_{\text {free }}$ & 0.2011 \\
Cruickshank DPI & 0.0662 \\
No. of non-H atoms & \\
$\quad$ Protein & 3694 \\
Ebselen $\dagger$ & 49 \\
Ligand & 54 \\
$\quad$ Water & 416 \\
R.m.s. deviations & \\
$\quad$ Bonds $(\AA)$ & 0.014 \\
Angles $\left({ }^{\circ}\right)$ & 1.856 \\
Average $B$ factors $\left(\AA^{2}\right)$ & \\
Overall & 29.4 \\
Protein & 27.9 \\
Ebselen & 31.7 \\
Ligand & 45.7 \\
Water & 40.3 \\
Ramachandran plot & \\
Favoured regions $(\%)$ & 98 \\
Additionally allowed $(\%)$ & 1.5 \\
Outliers $¥ \%)$ & 0.5 \\
\hline
\end{tabular}

$\dagger$ Ebselen attached to Cys141A had only one atom with two positions (the Se atom) Ebselen attached to Cys141B had every atom in two positions. There are $16 \mathrm{non}-\mathrm{H}$ atoms in ebselen. $\quad$ Lys 36 is the only residue (just) outside the allowed region in both subunits $\left(\varphi=-100^{\circ}, \psi=-110^{\circ}\right)$.

reduced. A partial Cys25-Cys125 disulfide is also observed in PDB entry 6gj0 (Kraft et al., 2018).

Previous research suggested that Cys218 is the primary reactive cysteine residue (Knowles et al., 1992), which is supported by the reduced inhibition of C218A mutant IMPase by ebselen (Singh et al., 2013). In the structure reported here (PDB entry 6zk0) and other human IMPase structures Cys218 is largely buried and therefore seems to be an unlikely target for modification, as it is unclear how ebselen would gain access. If the side chain of Cys 218 is modified by ebselen it would be likely to lead to a substantial reorganization of the protein structure; Asp220 coordinates an active-site metal ion.

From our structure, it appears that Cys141 is likely to be the primary binding site of ebselen. The sulfur group of this residue is exposed on the surface of IMPase (Fig. 2), and this residue is not in close proximity to another cysteine residue in either the monomeric or the dimeric form, and thus is unlikely to form a disulfide bond (PDB entry 6zk0). Cys141 is conserved in mammals (Knowles et al., 1992; Singh et al., 2013), and an analogous cysteine residue (Cys138) is present in Staphylococcus aureus IMPase. Given this level of conservation, it is probable that Cys141 is a functionally important residue in IMPase (Dutta et al., 2014).

Cys141 has previously been shown to be a reactive cysteine residue, as demonstrated by its affinity for the thiol probes pyrenemaleimide (Greasley et al., 1994) and $n$-ethylmaleimide (Knowles et al., 1992). In this structure, ebselen is in an openring conformation, with the Se atom forming a selenosulfide bond to the sulfur group of Cys141, which is consistent with 


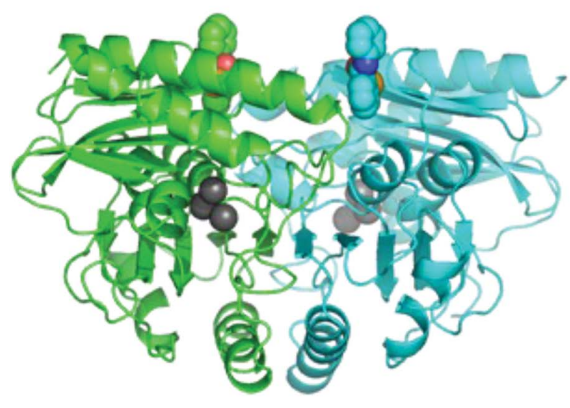

(a)

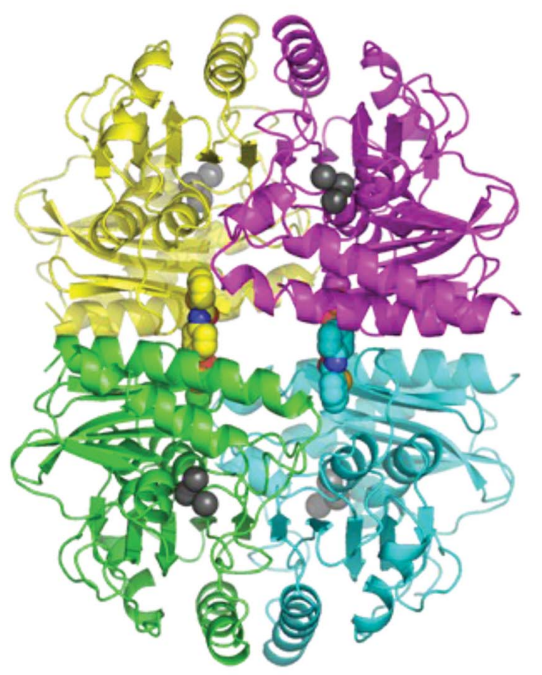

(d)

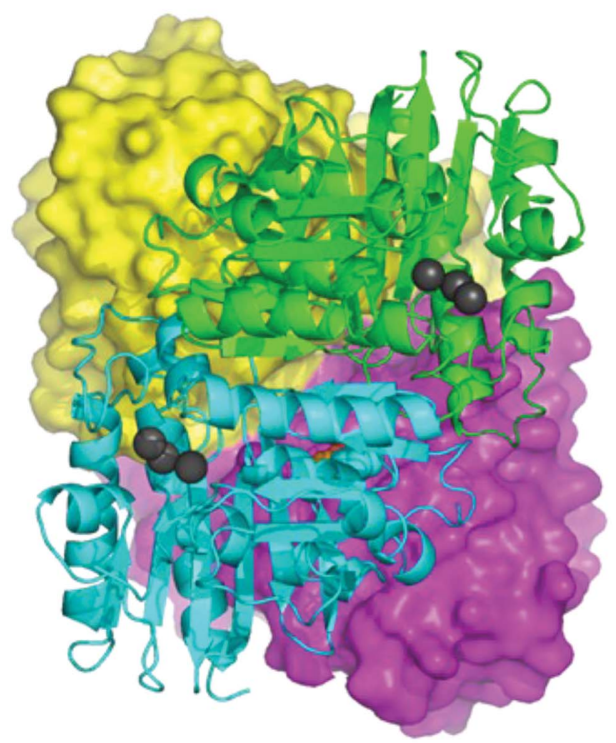

(g)

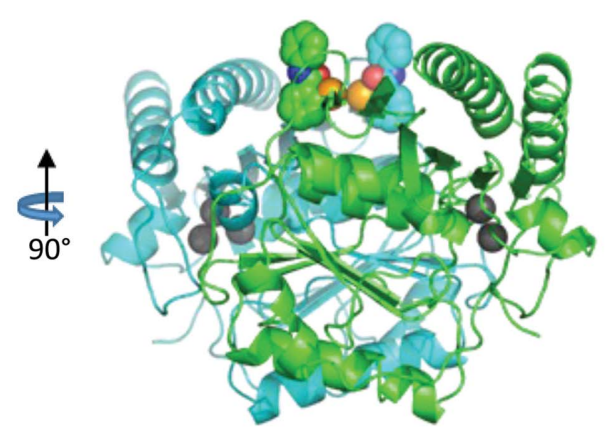

(b)

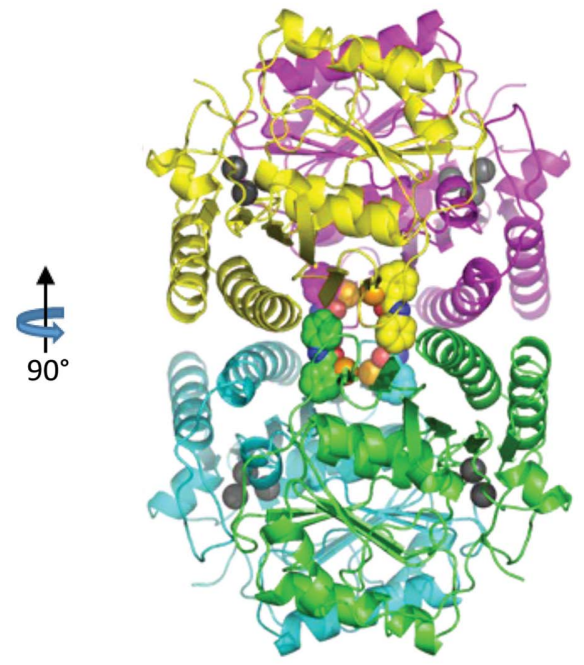

(e)

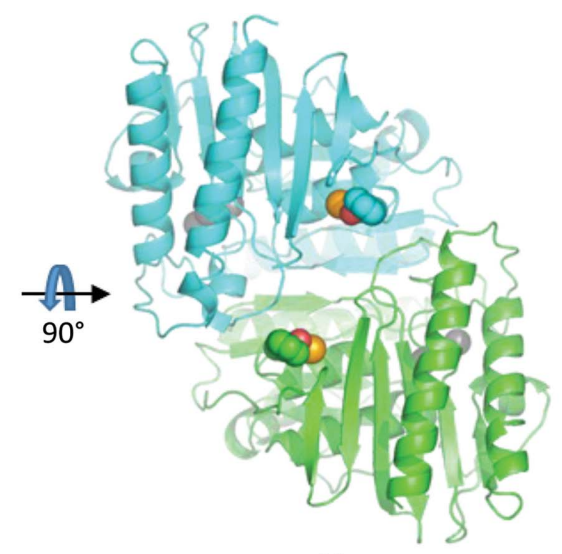

$(c)$

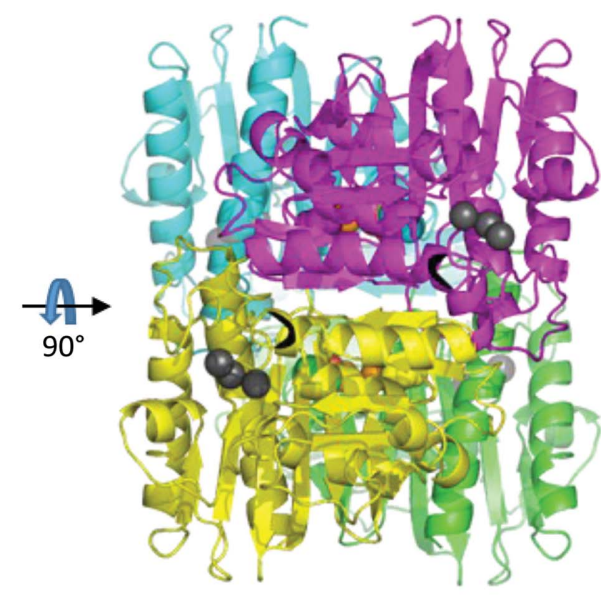

(f)

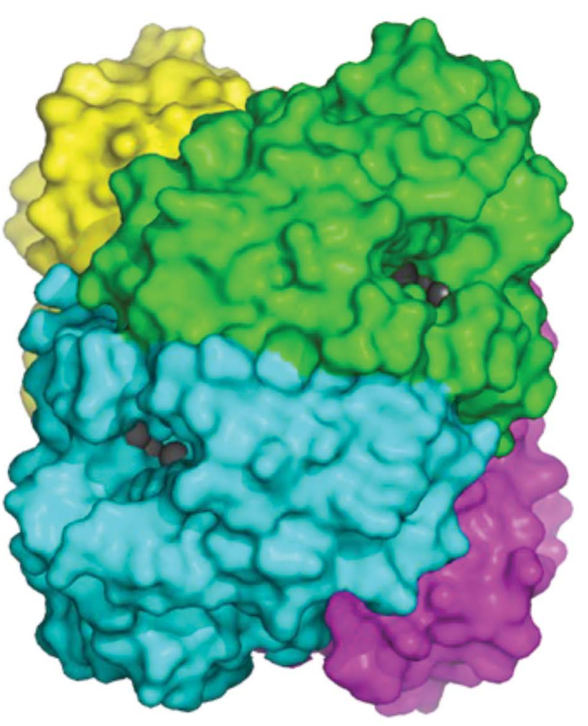

(h)

Figure 3

Orthogonal views of the IMPase dimer (and tetramer) showing ebselen on Cys141 and metal ions in the active sites based on the structure of PDB entry 6zk0. (a) The two subunits in the dimer are shown as green (subunit $A$ ) and cyan (subunit $B$ ) cartoons, with ebselen attached to Cys141A and Cys141B in space-filling representation. Metal ions $\left(\mathrm{Mn}^{2+} / \mathrm{Na}^{+}\right)$at each active site are shown as grey spheres. $(b, c)$ Orthogonal views. $(d, e, f)$ The same views as in $(a, b, c)$ but also showing a second dimer related by a crystallographic twofold axis. Subunit $A^{\prime}$ is in yellow and subunit $B^{\prime}$ is in magenta. The view in $(e)$ is along the crystallographic twofold that rotates the $A B$ dimer (green/cyan) onto the $A^{\prime} B^{\prime}$ dimer (yellow/magenta). ( $g, h$ ). Two views of the tetramer from underneath, showing that the three metal ions (grey/black spheres) at each active site are still accessible in the tetramer. In (h), a surface is shown for both dimers. 
the known binding mechanism of ebselen (Capper et al., 2018). Each monomer of IMPase in this structure has a single ebselen molecule bound to Cys141 (PDB entry 6zk0).

Whilst the conservation of Cys141 would suggest a critical role of this residue, its exact function remains unclear. The residue is not in close proximity to the active site, and therefore the residue is unlikely to be involved in catalytic activity. One possibility is that the residue may undergo post-translational modification in vivo and may be redox-active (Marino \& Gladyshev, 2012), linking ebselen to therapeutic effects as a known antioxidant.

\subsection{Ebselen-bound IMPase is observed to form a tetramer} with 222 symmetry in the solid state

Fig. 3 shows the position of Cys 141 on subunits $A$ and $B$ of an IMPase dimer. The two ebselen molecules covalently bind to each dimer and localize with two other ebselen molecules on a second dimer. The two ebselen molecules on each dimer increase the contacts with a neighbouring dimer, which gives a tetramer with approximate 222 symmetry in the crystal. However, as shown in Figs. 3( $g$ ) and 3(h), the active site still appears to be accessible.

Interestingly, other members of the IMPase superfamily, including fructose-1,6-bisphosphate (FBPase), have been observed to have both dimer and tetramer forms (Hines et al., 2007). Tetrameric forms of IMPase have also been observed in the anaerobic hyperthermophilic eubacterium Thermotoga maritima (Stieglitz et al., 2007).

\section{Discussion}

The IMPase crystal structure that is presented (PDB entry 6zk0) has ebselen covalently attached to Cys141; however, the extent to which this binding brings about the inhibitory effect of ebselen on IMPase is not clear. It is possible that the modification on Cys 141 is biologically relevant, and that this is a cysteine residue that is modified in vivo by ebselen, with binding over the previously suggested preferred residue Cys218 (Singh et al., 2013). Cys141 has been shown to be a reactive cysteine residue (Greasley et al., 1994), so it possible that ebselen binding at Cys141 causes inhibition of IMPase. However, the binding of ebselen does not affect the conformation of the active site or prevent dimer formation; with the high conservation of Cys141 across species, it is likely to be a functional residue with a potential regulatory redox role.

There is evidence that modulation of IMPase away from the active site and dimer interface can affect activity; synthetic peptides that disrupt IMPase-calbindin interactions prevent calbindin-mediated activation of IMPase (Noble et al., 2018) and mediate antidepressant-like effects in mice (Levi et al., 2013). It is possible that ebselen interferes with accessory protein binding, possibly by formation of the tetramer seen in the crystal, to moderate the activity of IMPase in vivo.

However, the possibility that the conditions used in crystallization do not reflect physiological conditions and that Cys 141 and Cys 218 could be modified differently by ebselen in vivo cannot be ruled out. The binding of ebselen to Cys 141 does not appear to have significantly altered the structure of the dimer or the active site. Should the ebselen/IMPase tetramers observed prove to be biologically relevant, this would suggest a new mechanism for the regulation and subsequent inhibition of IMPase that could be utilized in the development of novel therapeutics.

\section{Acknowledgements}

We are especially grateful to Dr Pierre Rizkallah and his colleagues for transporting and carrying out data collection on our crystals. We also thank Diamond Light Source Ltd (Didcot, UK) for access to synchrotron radiation on beamline I04. We thank Gareth Wright for helpful discussions.

\section{Funding information}

Funding for this project was provided by Cardiff University.

\section{References}

Atack, J. R., Broughton, H. B. \& Pollack, S. C. (1995). FEBS Lett. 361, $1-7$.

Azad, G. J. \& Tomar, R. S. (2014). Mol. Biol. Rep. 41, 4865-4879.

Berridge, M. J., Downes, C. P. \& Hanley, M. R. (1989). Cell, 59, 411419.

Brown, K. M. \& Tracy, D. K. (2013). Ther. Adv. 3, 163-176.

Capper, M. J., Wright, G. S. A., Barbieri, L., Luchinat, E., Mercatelli, E., McAlary, L., Yerbury, J. J., O'Neill, P. M., Antonyuk, S. V., Banci, L. \& Hasnain, S. S. (2018). Nat. Commun. 9, 1693.

Chantadul, V., Wright, G. S. A., Amporndanai, K., Shahid, M., Antonyuk, S. V., Washbourn, G., Rogers, M., Roberts, N., Pye, M., O'Neill, P. M. \& Hasnain, S. S. (2020). Commun. Biol. 3, 97.

Diederichs, K. (2006). Acta Cryst. D62, 96-101.

Dutta, A., Bhattacharyya, S., Dutta, D. \& Das, A. K. (2014). FEBS J. 281, 5309-5324.

Emsley, P. (2017). Acta Cryst. D73, 203-210.

Emsley, P., Lohkamp, B., Scott, W. G. \& Cowtan, K. (2010). Acta Cryst. D66, 486-501.

Evans, P. R. \& Murshudov, G. N. (2013). Acta Cryst. D69, 12041214.

Garman, E. F. (2010). Acta Cryst. D66, 339-351.

Geddes, J. R. \& Miklowitz, D. J. (2013). Lancet, 381, 1672-1682.

Gerstel, M., Deane, C. M. \& Garman, E. F. (2015). J. Synchrotron Rad. 22, 201-212.

Gill, R., Mohammed, F., Badyal, R., Coates, L., Erskine, P., Thompson, D., Cooper, J., Gore, M. \& Wood, S. (2005). Acta Cryst. D61, 545-555.

Greasley, P. J., Hunt, L. G. \& Gore, M. G. (1994). Eur. J. Biochem. 222, 453-460.

Harwood, A. J. (2005). Mol. Psychiatry, 10, 117-126.

Hines, J. K., Fromm, H. J. \& Honzatko, R. B. (2007). J. Biol. Chem. 282, 11696-11704.

Jin, Z., Du, X., Xu, Y., Deng, Y., Liu, M., Zhao, Y., Zhang, B., Li, X., Zhang, L., Peng, C., Duan, Y., Yu, J., Wang, L., Yang, K., Liu, F., Jiang, R., Yang, X., You, T., Liu, X., Yang, X., Bai, F., Liu, H., Liu, X., Guddat, L. W., Xu, W., Xiao, G., Qin, C., Shi, Z., Jiang, H., Rao, Z. \& Yang, H. (2020). Nature, 582, 289-293.

Knowles, M. R., Gee, N., McAllister, G., Ragan, C. I., Greasley, P. J. \& Gore, M. G. (1992). Biochem. J. 285, 461-468.

Kraft, L., Roe, S. M., Gill, R. \& Atack, J. R. (2018). Acta Cryst. D74, 973-978.

Levi, I., Eskira, Y., Eisenstein, M., Gilon, C., Hoffman, A., Tal-Gan, Y., Talgan, Y., Fanous, J., Bersudsky, Y., Belmaker, R. H., Agam, 
G. \& Almog, O. (2013). Eur. Neuropsychopharmacol. 23, 18061815.

Long, F., Nicholls, R. A., Emsley, P., Gražulis, S., Merkys, A., Vaitkus, A. \& Murshudov, G. N. (2017). Acta Cryst. D73, 112-122.

Marino, S. M. \& Gladyshev, V. D. (2012). J. Biol. Chem. 287, 44194425.

Masmaliyeva, R. C. \& Murshudov, G. N. (2019). Acta Cryst. D75, 505518.

Munnik, M. de, Lohans, C. T., Lang, P. A., Langley, G. W., Malla, T. R., Tumber, A., Schofield, C. J. \& Brem, J. (2019). Chem. Commun. 55, 10214-10217.

Murshudov, G. N., Skubák, P., Lebedev, A. A., Pannu, N. S., Steiner, R. A., Nicholls, R. A., Winn, M. D., Long, F. \& Vagin, A. A. (2011). Acta Cryst. D67, 355-367.

Nakamura, Y., Feng, Q., Kumagai, T., Torikai, K., Ohigashi, H., Osawa, T., Noguchi, N., Niki, E. \& Uchida, K. (2002). J. Biol. Chem. 277, 2687-2694.

Noble, J. W., Almalki, R., Roe, S. M., Wagner, A., Duman, R. \& Atack, J. R. (2018). Acta Cryst. D74, 1008-1014.

Phiel, C. J. \& Klein, P. S. (2001). Annu. Rev. Pharmacol. Toxicol. 41, 789-813.

Rybakowski, J. K. (2016). Curr. Alzheimer Res. 13, 887-893.
Singh, N., Halliday, A. C., Thomas, J. M., Kuznetsova, O. V., Baldwin, R., Woon, E. C. Y., Aley, P. K., Antoniadou, I., Sharp, T., Vasudevan, S. R. \& Churchill, G. C. (2013). Nat. Commun. 4, 1332.

Singh, N., Sharpley, A. L., Emir, U. A., Masaki, C., Herzallah, M. M., Gluck, M. A., Sharp, T., Harmer, C. J., Vasudevan, S. R., Cowen, P. J. \& Churchill, G. C. (2015). Neuropsychopharmacology, 41, 1768-1778.

Stieglitz, K., Roberts, M., Li, W. \& Stec, B. (2007). FEBS J. 274, 24612469.

Ullrich, V., Weber, P., Meisch, F. \& von Appen, F. (1996). Biochem. Pharmacol. 52, 15-19.

Weik, M., Bergès, J., Raves, M. L., Gros, P., McSweeney, S., Silman, I., Sussman, J. L., Houée-Levin, C. \& Ravelli, R. B. G. (2002). J. Synchrotron Rad. 9, 342-346.

Williams, R. S., Cheng, L., Mudge, A. W. \& Harwood, A. J. (2002). Nature, 417, 292-295.

Winter, G. (2010). J. Appl. Cryst. 43, 186-190.

Winter, G., Lobley, C. M. C. \& Prince, S. M. (2013). Acta Cryst. D69, 1260-1273.

Wojdyr, M., Keegan, R., Winter, G. \& Ashton, A. (2013). Acta Cryst. A69, s299.

Won, E. \& Kim, Y. (2017). Int. J. Mol. Sci. 18, 2679. 